\title{
Improving miRNA-mRNA interaction predictions
}

\author{
Daniel Tabas-Madrid ${ }^{1 \dagger}$, Ander Muniateguii ${ }^{2+}$, Ignacio Sánchez-Caballero ${ }^{1}$, Dannys Jorge Martínez-Herrera ${ }^{1}$, \\ Carlos Oscar S Sorzano ${ }^{1}$, Angel Rubio ${ }^{2^{*}}$, Alberto Pascual-Montano ${ }^{1 *}$
}

From The 25th International Conference on Genome Informatics (GIW/ISCB-Asia)

Tokyo, Japan. 15-17 December 2014

\begin{abstract}
Background: MicroRNAs are short RNA molecules that post-transcriptionally regulate gene expression. Today, microRNA target prediction remains challenging since very few have been experimentally validated and sequencebased predictions have large numbers of false positives. Furthermore, due to the different measuring rules used in each database of predicted interactions, the selection of the most reliable ones requires extensive knowledge about each algorithm.
\end{abstract}

Results: Here we propose two methods to measure the confidence of predicted interactions based on experimentally validated information. The output of the methods is a combined database where new scores and statistical confidences are re-assigned to each predicted interaction. The new scores allow the robust combination of several databases without the effect of low-performing algorithms dragging down good-performing ones. The combined databases obtained using both algorithms described in this paper outperform each of the existing predictive algorithms that were considered for the combination.

Conclusions: Our approaches are a useful way to integrate predicted interactions from different databases. They reduce the selection of interactions to a unique database based on an intuitive score and allow comparing databases between them.

\section{Background}

MicroRNAs (miRNAs) are a novel class of endogenous, $\sim 22 \mathrm{nt}$ long RNAs that post-transcriptionally regulate gene expression [1]. They guide the RNA-induced silencing complex (RISC) to their mRNA targets by sequence complementarity. In animals, miRNAs generally bind to the 3'UTR of the mRNA imperfectly and in most cases lead to translational inhibition of its targets [2]. In plants, most miRNAs match perfectly to the coding region of their targets causing mRNA degradation [3]. However, some other interactions have been identified in vitro, e.g. interactions with the 5'UTR and with the coding region of transcripts in mammals, with the 3'UTR of plants transcripts and even some expressionenhancing miRNAs[4].

\footnotetext{
* Correspondence: arubio@ceit.es; pascual@cnb.sic.es

+ Contributed equally

${ }^{1}$ National Center for Biotechnology-CSIC. Darwin 3. 28049, Madrid, Spain

${ }^{2}$ CEIT and TECNUN, University of Navarra, San Sebastián, Spain
}

Full list of author information is available at the end of the article
MiRNAs are known to be involved in development [1], cell proliferation [5] and differentiation [6], apoptosis [7], cell cycle progression [8], tumorigenesis[9], and many other physiological and pathological processes[10].

There are several experimentally defined rules of miRNA targeting in mammals. Sequence complementarity with the "seed", generally the nucleotides 2 to 7 , is sufficient to produce the repression of most animal mRNAs $[5,11]$. The seed matches are grouped into four canonical types: 6 mer, $7 \mathrm{mer}-\mathrm{m} 8,7 \mathrm{mer}-\mathrm{A} 1$ and $8 \mathrm{mer}$. There are also other features outside this region. In fact, sequence complementarity to nucleotides 13-16 of the miRNA can either enforce the affinity (supplementary pairing) or compensate for an incomplete seed pairing (complementary pairing). Furthermore, G:U wobbles within the seed are unfavorable to the regulation by miRNAs[12]. The thermodynamic stability of the duplex is a crucial feature of the interaction [13]. There are few other specificities for the mode of action of miRNAs in plants and metazoa[14]. 
Development of deep-sequencing methods have increased considerably the number of newly discovered miRNAs[15]. MirBase[16] is up to date the most complete database of precursor and mature miRNAs. Its latest update (release 20, June 2013) covers 206 species and contains 30,424 precursors and 24,521 microRNAs.

Presently there is a plenty of algorithms and databases to predict miRNA-mRNA interactions based on sequence, physical-chemistry properties, expression levels or even experimental validations. For a common molecular biologist this panorama represents yet another level of complexity to its every day work since there is no single answer to the questions of what are the target genes for a single miRNA? Which prediction algorithms or databases is the best performing one? How can I reduce the number of predicted targets? The nature of the questions reflects the current panorama. In this work we try to provide answers to those questions by proposing a methodology that combines and re-score the miRNA-mRNA interactions from all different available sources. Our intention is to provide the community with a unique source for miRNA-mRNA interactions based on the goodness of all available ones.

\section{miRNA-mRNA interactions}

Experimentally validated interactions. Currently there are several databases with experimentally-validated interactions such as: miRWalk[17], miRecords[18], TarBase [19], miRTarBase[20] and starBase[21]. They differ mainly on the number of interactions they host. The starBase database includes interactions validated only by HITSCLIP and by Degradome Sequencing. These techniques provide more accurate information about direct miRNAmRNA interactions, and also about the exact binding site [22]. Table 1 contains detailed information about databases and number of interactions per organism.

Computationally predicted interactions. Today, the use of computational methods has sped up considerably miRNA target analysis. Currently available computational methods can be grouped into ab initio, machine learning and hybrid methods [15].

Table 1 Number of experimentally validated interactions.

\begin{tabular}{ccccc}
\hline & Mirtarbase & Tarbase & Mirwalk & Mirecords \\
\hline Caernorhabditis elegans & 30 & - & - & 17 \\
Drosophila melanogaster & 115 & - & - & 81 \\
Danio rerio & 102 & - & - & 32 \\
Gallus gallus & 16 & - & - & - \\
Homo sapiens & 2860 & 878 & 5668 & 1276 \\
Mus musculus & 537 & 70 & 2749 & 194 \\
Rattus norvegicus & 231 & - & 1514 & 39 \\
\hline
\end{tabular}

Summary of the number of validated interactions for each species we have studied as well as the source where this interaction was reported.
$A b$ initio algorithms are based on the experimentally defined rules of miRNA targeting. Among them, MiRanda [23] uses an estimated complementarity score to select the duplexes, MicroTar[24] considers different sequence complementarities in the seed (nt 1-7 and nt 2-8) allowing for G:U wobbles. PITA[13] selects seed matches of six to eight nucleotides, allowing up to one G:U wobble in 7 and 8-mers and up to one mismatch in 8-mers. TargetScan [11] first searches perfect complementarities to the seed and then calculates a score, based on the site type, local A-U enrichment and other aspects of the seed match context. Finally, FindTar[25] finds seed matches allowing up to one G:U wobble and scores them by the position of the central loop. Except TargetScan, these methods consider the thermodynamic stability of duplexes using Vienna RNA package [26]. For instance, RNAhybrid[27] and miRiam[28], first maximize the thermodynamic stability of the miRNA-mRNA pair and then search for sequence complementarities.

Machine learning algorithms, such as RFMirTarget [29] and MultiMiTar[30], filter predictions from $a b$ initio algorithms by using classifiers trained with feature patterns extracted from experimentally-validated interactions. RFMirtarget is based on a random forest classifier that evaluates 17 features extracted from a previous prediction performed using miRanda on the test dataset. MultiMiTar is a support vector machine-based algorithm that rewards 90 features of the miRNA-mRNA pair. These features are selected by means of the novel multiobject metaheuristic technique AMOSA[31] integrated with SVM. Both methods were trained 289 interactions extracted from miRecords database and 289 systematically identified tissue-specific negative examples and evaluated using an independent experimentally validated set of interactions.

One example of hybrid methods is NBmiRTar algorithm [32]. It first applies the miRanda algorithm, and then uses a Näive Bayes classifier to filter the output based on 57 features. NBmiRTar was trained with a set of 225 positive miRNA targets of 5 animal species and 38 negative interactions from TarBase.

Databases of predicted interactions. Some computationally predicted interactions have been incorporated to different databases: EIMMo[33], DIANA-microT[34], Microcosm [35], http://Microrna.org[36], TargetScan [37], MirDB[38], PITA, miRWalk-predictive [17] and TargetSpy.

Among them, MiRWalk algorithm first searches for perfect complementarities in the seed and then extends it until a mismatch is found. EIMMo searches possible target sites of a microRNA in four different species, retrieves the number of species in which the site is conserved and using Bayes statistics it calculates the probability of conservation of the seed. DIANA-microT 
searches for 7-, 8- or 9-nt long seed-matches, or 6-nt seeds with one G:U wobble and retrieves the weighted sum of conserved and non-conserved sites of a gene in up to 27 species. Finally, MirDB uses the SVM-based algorithm MirTarget2 [39]. A comparative description of in silico prediction methods is shown in Table 2.

\section{Combination of miRNA-mRNA interactions}

One drawback of sequence-based methods is the large numbers of false positives they predict. Some studies have made use of conservation analysis for interaction filtering. However, this can lead to the loss of speciesspecific interactions.

In the last years, several unions and intersections of different databases have been proposed to improve the specificity and sensitivity of the predictions. One of these attempts was done in [40]. Here the performance of TargetScan, DIANA-microT, miRanda, TargetScanS and PicTar[41], as well as their combinations was compared. The highest value of specificity was obtained for the intersection of the five algorithms and the specificity for the different proposed combinations was over $66.7 \%$.

In [42], authors experimentally analyzed the intersection of the possible microRNA regulators predicted by TargetScan, miRanda and PicTar for the human angiotensin II type 1 receptor (hAT1R). They validated one of the initially considered interactions showing that using the intersection of databases was a viable way of interaction filtering.

Other approaches, such as, ComiR[43], ExprTarget [44], Ranking Aggregation[45], BcmicrO[46], GenMiR3 [47] and a Bayesian Graphical model[48], combine the scores of different databases (see Table S1 in additional file 1).

The aim of GenMiR3 and the Bayesian Graphical model is not to combine different databases but to extract the most outstanding interactions given the miRNA and mRNA expression data as well as sequence based information. However both perform database combination internally and are based on logistic priors.

Ranking Aggregation method is designed to combine different numbers of top- $\mathrm{K}$ ranked lists and is based on Cross Entropy Monte Carlo method. It was successfully evaluated in combining the ranked list of targets of human miR-155-5p predicted by miRanda, TargetScan and PicTar.

ComiR combines four databases by estimating the probability of every gene of being targeted by the input set of miRNAs by using an SVM algorithm. If available, miRNA expression data is also considered.

ExprTarget uses a logistic regression to combine the scores of different databases with expression data of mRNAs and miRNAs. In their model, each of the scores of the databases is weighted by means of their capability of replicating experimentally validated interactions. Expression values are used to fit a linear model for each pair and the obtained p-value of the fit is used as an additional score in the model. ExprTarget is based on miRanda, TargetScan and PicTar scores. TarBase is used as gold standard. ROC curves [49] showed that ExprTarget outperforms individual databases.

Finally, $\mathrm{BCmicrO}$ uses a probabilistic model to determine how likely is an interaction to be experimentally validated given the scores in different databases. This model is expressed in terms of individual conditional probabilities, one per database and interaction. The authors generated a negative set of miRNA-targets to use as true negatives. This method was tested with TargetScan, miRanda, Pictar, mirTarget, PITA and DIANA-microT. ROC curves showed BCmicrO method outperformed individual databases.

Our approaches. Currently, there is no method considerably better than others in predicting microRNA targets. Some recently developed tools provide different ways to combine predictions of several algorithms, assuming that they perform similarly and share the same scoring system, which is not necessarily true. Ideally, an integration of different prediction algorithms should take into account their level of performance as well as the score of each interaction when it is reported by more than one method. In this study, we introduce two complementary approaches to improve the miRNA-mRNA interactions by combining nine predictive algorithms, as well as experimentally validated interactions. Global performance of the algorithms as well as the individual score of every interaction reported by different methods is taken into account. We show that the combination outperforms previous approaches while reducing the number of potential targets candidates.

\section{Results and discussion}

Reliability of databases. It is difficult to compare across the different databases of interactions since they differ in size, quality of the interactions and the ability of the scores to reflect the quality of the interactions. We have used a hypergeometric test to measure the reliability of databases (see section "Measuring the reliability of databases" in the Materials and Methods section). Results are shown in Table 3.

This table shows the different databases sorted by $\mathrm{z}$ score that, in turn, is a measure of their enrichment in experimentally-validated interactions when sorted by their provided score. The first two rows of this table correspond to WSP and LRS databases in this work and we will refer to them later. EiMMo and DIANA-microT are top-ranked according to the z-score. TargetScan, although being lowly ranked by the $\mathrm{z}$-score, has the highest proportion of experimentally validated interactions. It seems that TargetScan focuses on including 
Table 2 Comparison of sequence-based algorithms for miRNA-mRNA target predictionn.

\begin{tabular}{|c|c|c|c|c|c|c|c|c|c|c|c|c|}
\hline Method $^{\mathrm{a}}$ & Name & Seed & $\Delta \mathrm{G}$ & Conserv. & Wobbles & $\Delta \Delta \mathrm{G}$ & Other features & $\begin{array}{l}\text { Type of } \\
\text { classifier }\end{array}$ & Scoring & $\mathrm{DB} ?$ & software? & website \\
\hline Al & miRanda & $\checkmark$ & $\checkmark$ & $\checkmark$ & & & $\begin{array}{l}\text { matches with the first } 11 \mathrm{nt} \text { 's of the miRNA are } \\
\text { rewarded }\end{array}$ & & score & $\checkmark$ & $\checkmark$ & http://www.microrna.org \\
\hline $\mathrm{Al}$ & TargetScan & $\checkmark$ & & $\checkmark$ & & & different seed types and AU content & & score & $\checkmark$ & $\checkmark$ & http://www.targetscan.org \\
\hline $\mathrm{Al}$ & PicTar & $\checkmark$ & $\checkmark$ & $\checkmark$ & & & & & score & $\checkmark$ & & http://pictar.mdc-berlin.de/ \\
\hline Al & RNA22 & $\checkmark$ & $\checkmark$ & & & & $\begin{array}{l}\text { miRNA paired to statistically significant patterns in the } \\
\text { mRNA }\end{array}$ & & & & & $\begin{array}{l}\text { http://omictools.com/ } \\
\text { rna22-s5063.html }\end{array}$ \\
\hline Al & RNAhybrid & $\checkmark$ & $\checkmark$ & & & & MFEs modeled as extreme-value distributed & & MDE (energy) & & $\checkmark$ & $\begin{array}{l}\text { http://bibiserv.techfak.uni- } \\
\text { bielefeld.de/rnahybrid/ }\end{array}$ \\
\hline \multirow[t]{2}{*}{$\mathrm{Al}$} & PITA & $\checkmark$ & $\checkmark$ & & $\checkmark$ & $\checkmark$ & 1) G:U allowed in 7mer seed & & score & $\checkmark$ & $\checkmark^{b}$ & $\begin{array}{l}\text { http://genie.weizmann.ac. } \\
\text { il/pubs/mir07/ }\end{array}$ \\
\hline & & & & & & & 2) G:U, 1 mismatch allowed in 8mer & & & & & \\
\hline $\mathrm{Al}$ & EiMMo & $\checkmark$ & & $\checkmark$ & & & $\begin{array}{l}\text { model the evolution of orthologous target sites in } \\
\text { related species }\end{array}$ & & score & $\checkmark$ & & $\begin{array}{c}\text { http://www.mirz.unibas.ch/ } \\
\text { ElMMo3/ }\end{array}$ \\
\hline $\mathrm{Al}$ & $\begin{array}{l}\text { DIANA- } \\
\text { microT }\end{array}$ & $\checkmark$ & $\checkmark$ & $\checkmark$ & $\checkmark$ & & & & score & $\checkmark$ & & $\begin{array}{l}\text { http://diana.cslab.ece.ntua. } \\
\text { gr/microT/ }\end{array}$ \\
\hline $\mathrm{Al}$ & MicroTar & $\checkmark$ & $\checkmark$ & & & & & & $p$-value & & $\checkmark$ & $\begin{array}{l}\text { http://tiger.dbs.nus.edu.sg/ } \\
\text { microtar }\end{array}$ \\
\hline Al & FindTar & $\checkmark$ & $\checkmark$ & & $\checkmark$ & & central loop score to reduce false positives & & score and energy & & $\checkmark$ & $\begin{array}{l}\text { http://bio.sz.tsinghua.edu. } \\
\text { cn/ }\end{array}$ \\
\hline $\mathrm{Al}$ & miRiam & $\checkmark$ & $\checkmark$ & & & $\checkmark$ & & & & $\checkmark$ & $\checkmark$ & $\begin{array}{c}\text { http://ferrolab.dmi.unict.it/ } \\
\text { miriam.html }\end{array}$ \\
\hline $\mathrm{Al}$ & microcosm & $\checkmark$ & $\checkmark$ & $\checkmark$ & & & $\begin{array}{l}\text { Uses miRanda. Requires: complete seed } \\
\text { complementarity and conservation at the same } \\
\text { position and in } \geq 2 \text { species }\end{array}$ & & score & $\checkmark$ & & $\begin{array}{l}\text { http://www.ebi.ac.uk/ } \\
\text { enright-srv/microcosm/ } \\
\text { htdocs/targets/v5/ }\end{array}$ \\
\hline Al & miRWalk & $\checkmark$ & $\checkmark$ & & & & $\begin{array}{l}\text { also a DDBB with experimentally-validated targets } \\
\text { from text mining }\end{array}$ & & $p$-value & & & http://mirwalk.uni-hd.de/ \\
\hline ML & miTarget & $\checkmark$ & $\checkmark$ & & & & Starting set: miRanda. Radial basis function & SVM & & & & $\begin{array}{l}\text { http://cbit.snu.ac.kr/ } \\
\sim \text { miTarget/introduction. } \\
\text { html }\end{array}$ \\
\hline ML & MirTarget2 & $\checkmark$ & $\checkmark$ & $\checkmark$ & & & Initial set: TargetScan, PicTar, miRanda, MirTarget & SVM & $\begin{array}{l}\text { score (from } \\
\text { probabilities) }\end{array}$ & $\checkmark$ & & http://mirdb.org \\
\hline$M L$ & TargetSpy & $\checkmark$ & $\checkmark$ & $\checkmark$ & & & $\begin{array}{c}\text { Starting set: PicTar. Generates candidate zones of } \\
\text { binding and a representative hybrid (1st or 2nd nt of } \\
\text { the miRNA is paired) }\end{array}$ & & score & $\checkmark$ & $\checkmark$ & http://www.targetspy.org \\
\hline ML & mirSVR & $\checkmark$ & $\checkmark$ & $\checkmark$ & & & Starting set: miRanda & SVR & $\begin{array}{l}\text { mirSVR score } \\
\text { (probability for } \\
\text { down-regulation) }\end{array}$ & $\checkmark$ & $\checkmark$ & http://microrna.org \\
\hline $\mathrm{H}$ & NBmiRTar & $\checkmark$ & $\checkmark$ & $\checkmark$ & & & $\begin{array}{l}\text { NB classifier is applied to the output of the miRanda } \\
\text { program }\end{array}$ & $\begin{array}{l}\text { Naïve } \\
\text { Bayes }\end{array}$ & $\begin{array}{l}\text { NB score } \\
\text { (probability) }\end{array}$ & & & \\
\hline a & $\mathrm{Al}=\mathrm{Ab} \operatorname{Initic}$ & $\mathrm{O}, \mathrm{ML}$ & $=\mathrm{Ma}$ & chine Lea & hing, $\mathrm{H}=\mathrm{H}$ & ybrid & & & & & & \\
\hline$b$ & Academic us & se only & & & & & & & & & & \\
\hline
\end{tabular}

Comparison of different algorithms of miRNA-mRNA target prediction including different algorithm features, the databases and software availability, scoring method, type of classifier, and species for which the algorithm was designed. 
Table 3 Reliability of databases.

\begin{tabular}{|c|c|c|c|c|c|c|}
\hline & (1) & (2) & (3) & (4) & (5) & (6) \\
\hline Method & $Z_{\text {score }}$ & $\#$ int. $Z_{\text {score }}$ & \# DDBB & \# EV & \# EV / \# DB & $\%$ drawn \\
\hline LRS & -89.27 & 163829 & 4669137 & 4286 & $9.18 \mathrm{E}-04$ & 9.2 \\
\hline WSP & -84.52 & 123589 & 4669137 & 4286 & $9.18 \mathrm{E}-04$ & 6.94 \\
\hline EiMMo & -61.87 & 191582 & 1781671 & 2949 & $1.66 \mathrm{E}-03$ & 10.75 \\
\hline DIANA-microT & -54.51 & 269525 & 2889574 & 3010 & $1.31 \mathrm{E}-03$ & 11.77 \\
\hline http://www.microrna.org & -21.2 & 134227 & 737379 & 2685 & $3.64 \mathrm{E}-03$ & 18.2 \\
\hline microcosm & -17.99 & 6035 & 352016 & 784 & $2.23 \mathrm{E}-03$ & 1.71 \\
\hline PITA & -15.2 & 75683 & 206722 & 1425 & 6.89E-03 & 36.61 \\
\hline TargetSpy & -14 & 178114 & 300000 & 653 & $2.18 \mathrm{E}-03$ & 59.37 \\
\hline miRWalk & -9.92 & 422089 & 780000 & 1243 & $1.59 \mathrm{E}-03$ & 54.11 \\
\hline TargetScan & -9.29 & 19491 & 132809 & 1832 & $1.38 \mathrm{E}-02$ & 14.68 \\
\hline mirTarget & -5.08 & 149088 & 691265 & 234 & 3.39E-04 & 21.57 \\
\hline
\end{tabular}

(1) Minimum z-score of a hypergeometric distribution. The lower the z-score the more statistically significant the enrichment in experimentally validated interactions is. (2) Number of interactions for the minimum z-score. (3) Total number of interactions in the database. (4) Number of experimentally validated (EV) interactions in the database. (5) Proportion of EV interactions within the database. (6) Proportion of selected interactions in the database for the minimum zscore.

only the most reliable interactions. On the other hand, mirTarget is a medium-sized database, but the quality of interactions, in terms of proportion of experimentally validated interactions, is small, and therefore, lowly ranked in this table. TargetScan results indicate that using $\mathrm{z}$-score as the unique parameter for database comparison is not sufficient (other factors should also be considered, for example, column (6) of table 3). However, this measurement seems reasonable to compare databases with large non-uncut lists of interactions. This is the case of many of the databases used in this work.

Comparison of methods. Materials and methods describe in detail the two approaches we propose to combine predictive miRNA-mRNA interactions from nine different algorithms widely used by the scientific community. The first one, named Weighted Scoring by Precision (WSP) is based on summing up the weighted scores for different databases whilst the second one applies logistic regression to find the combined score (LRS). These approaches were evaluated using four different experimentally validated interactions databases to define the tradeoff between sensitivity and specificity. The evaluation of our methods has been restricted to the comparison against the predicted databases and algorithms and compared with two widely used integration methods: the union and the intersection.

Both approaches outperform any of the predicting algorithms. A first evaluation of the predictions of our two methods has been done using the hypergeometric test used previously for database reliability measurement (Table 3). It can be noted that both of them rank better than any other considered database in terms of z-score and number of interactions. Figure 1 shows the ROC curve of the individual predicting algorithms as well as the two combined approaches we introduced here. LRS outperforms the rest of the algorithms in terms of the ROC curve, while WSP also improves almost all the other algorithms and behaves similarly to EIMMO. It is important to notice that the number of interactions predicted by the different algorithms is quite different, except for the combined approaches that use all miRNAmRNA pairs reported by the different methods (see Table 3).

For this particular application, both, the number of False Positives and True Negatives, cannot be exactly estimated. To limit the effect of this missing information, we proposed the use of the precision curve described in Figure 2. The ranking of the different methods resemble those reported in the ROC curve, however, the improvement in performance of the combined approaches is now clearer. Figure 1 shows an example of this effect is ROC AUC of the intersection (0.91), it is much higher than those of the proposed methods (i.e., 0.79 for WSP and 0.84 for LRS). It appears that the intersection is the best-performing method in terms of ROC AUC. This appreciation is misleading. Intersection seems to be the best method since it is the most conservative one. Intersecting all the prediction databases results in only 117 interactions and most of them are obviously experimentally validated. The ROC curve is also misleading because since there are no databases containing non-interactive miRNA-mRNA pairs, the number of False Positives and True Negatives cannot be exactly estimated. There is no optimal solution available for this and that is why we proposed the use of the precision curve described in Figure 2. As can be seen in Figure 2, the intersection is not the most prominent. On the other hand, it is known that if the positive and negative populations are not evenly balanced, the ROC curve does not reflect adequately the behavior of the classifier[49]. 


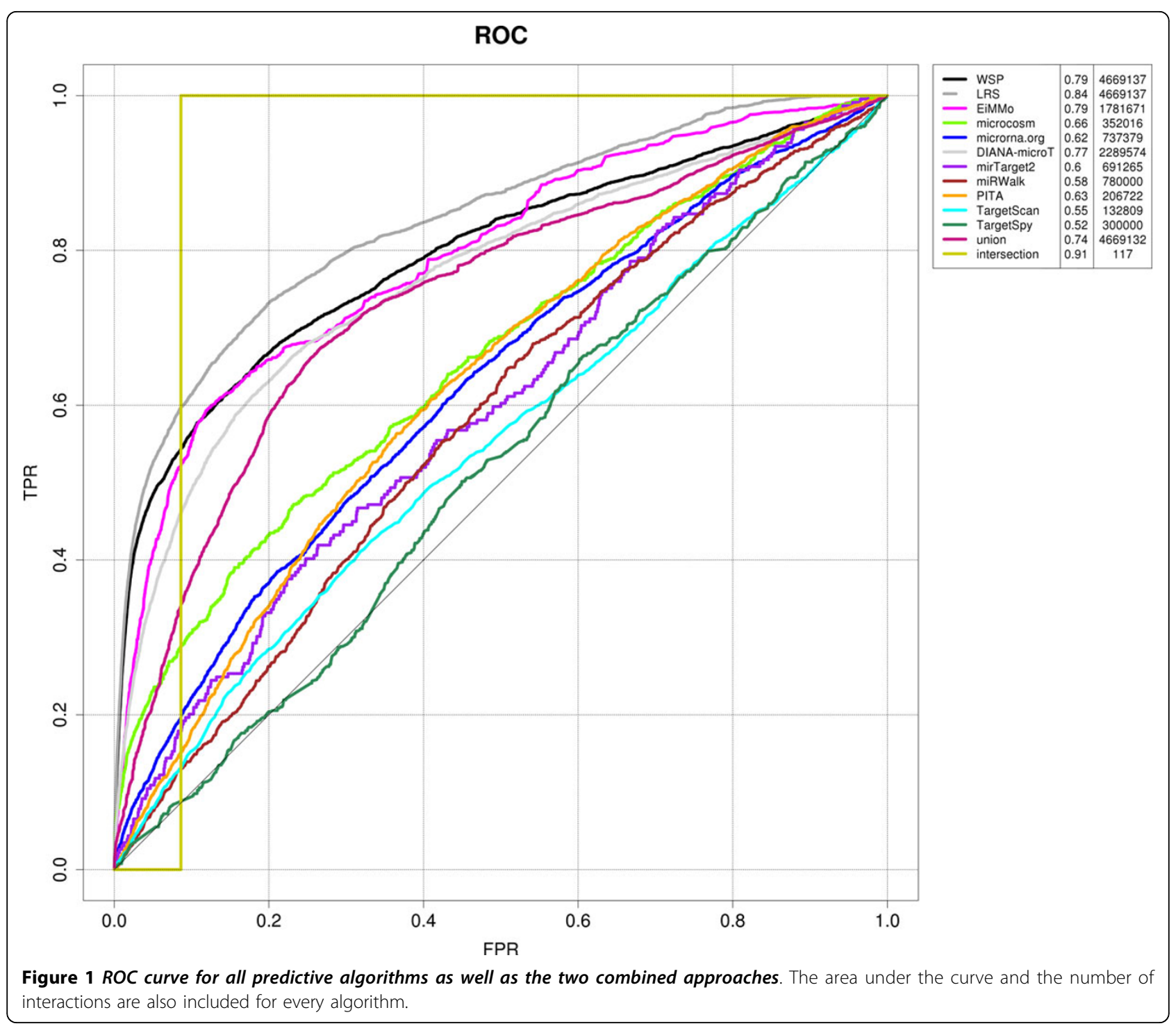

Differences between the two proposed approaches are apparent in both the ROC curve and the Precision curves. We noticed, however, that the main inconsistencies concern the first 400 interactions, which represents less than $0.01 \%$ of the entire universe of predicted miRNA-mRNA pairs. This is a very small percentage of interactions. Our observation is that the exact score values in the method are not as important as being in the top of the list with a high prediction value. From that perspective, both methods are perfectly compatible. These differences are highlighted in Figure S2 in the supplementary material.

In this work we have compared our methods with the two most used integration and straightforward approaches: the union and the intersection. Although a full comparison with all available methods would be ideal, this is not always possible for several reasons: a) The idea in this contribution is to use the largest amount of individual prediction methods and databases available and therefore the integration needs to be performed with the same databases and algorithms to make a fair comparison. Most of the integration approaches that we cite in the paper use only a subset of the databases and this would make the comparison very unfair. b) Availability of the code or data: most of these methods do not provide a full code we can run and modify or the full interactions data; therefore, a full comparison is in some cases virtually impossible. c) Lack of simple ways to reproduce and calculate these results several times.

Despite of our efforts in making a full comparison, only ExprTarget could be included, even if the comparison was not totally equal in the conditions we have used for the union and intersection. Supplementary section 4 contains all details. 




Figure 2 Precision curve for all predicting algorithms and the combination methods. The curve was corrected by subtracting the precision value that corresponds to random interactions. The $Y$ axis shows the Precision and the $\times$ axis the interactions sorted by score in descending order.

We consider that, as it is already happening in other areas, there is a need of community efforts to provide the data, and algorithms available to facilitate comparison.

All the interactions from the computational methods described in this work, as well as all the experimentally validated interactions are available to the scientific community in a database accessible via web at http://m3rna. cnb.csic.es. Predictions are sorted according the criteria presented here. Each individual prediction is reported with a new combined score. This functionality allows researchers to access a unified repository with most of the available and known information about miRNA-mRNA interactions and use it to compare it with their own methods.

\section{Conclusions}

No miRNA-mRNA algorithm makes perfect predictions under every condition. Because of the multi-faceted nature of miRNA targeting, and the lack of consensus among existing predictions, it makes sense to combine them in a way that maximizes the number of true predicted results while minimizing that of false ones. There have been previous attempts to combine the predictions of several algorithms by first taking their union or intersection, as a way to improve accuracy or coverage, balancing out their sensitivity and specificity, and finally, choosing the most likely candidates by consensus. Most of these approaches give the freedom to choose which combination of prediction algorithms to use. The main issue is that a significant proportion of users do not have the necessary information about each algorithm's performance to make an educated decision.

Our approaches present alternative solutions to this problem by assigning confidence scores to each prediction regardless the algorithm that originally predicts it. Both methods provide a score that objectively quantifies the quality of a particular interaction given its score and the database (or databases) that predict it. This solves the implicit problem of choosing a candidate by consensus in which the confidence of the predictions is not taken into account. In addition, it solves the problem of setting the thresholds (different for each of the prediction databases) to decide whether a predicted interaction is sufficiently reliable or not.

There are some limitations in our approaches that represent open research problems for the scientific community and could be interesting future research directions. For example, we assume that prediction algorithms have a high precision when they contain many validated interactions in their top scores, but this does not necessarily mean that algorithms with low precision are not predicting true interactions. It may just mean that the interactions they predict are harder to prove experimentally, or because the necessary experiments to validate them were never carried out. Almost all prediction 
algorithms, however, make this assumption. Another weak point in our approaches is that they start with the predictions that were reported by their authors and that are publicly available. It turns out that different reported interaction databases use different versions of sequence databases and therefore, the universe of mRNA used for predictions by the different algorithms is not exactly the same. Rerunning all prediction algorithms with the same mRNA and miRNA sequences would solve this limitation. It is difficult, however, to reproduce the same results than the ones reported on the authors' web sites because of parameters selection and availability of the code. This issue has no simple solution and no statistical tests or algorithms would solve it. We consider that a community effort by the algorithms' providers is the only way to solve this problem, either by making all codes available or by providing updated results of their prediction based on a common set of miRNAs and mRNAs. Our methods presented here do the best they can with the available information and helps in minimizing the negative effect of this lack of homogeneity of the databases. As a final limitation, the lack of information at the transcript level from both predicting algorithms and experimentally validated databases create an important limitation to any method that combines predicting algorithms. Interactions make more sense when they are described at the transcript level, if possible.

As our understanding of miRNA targeting improves and experimental methods become cheaper and more precise, our combined database will become more sensitive and specific. A good example is the starBase database [21] that contains interactions identified by the latest and more precise high-throughput techniques. It will certainly become one of the reference databases for experimentally validated miRNA-mRNA interactions. Integrating starBase, as well as any other new database, will be a future logical continuation of this work and a good input for future versions of $\mathrm{M}^{3}$ RNA. Besides the databases of experimentally validated miRNA-target interactions, there are quite a few large-scale expression-based analyses which can also be used as alternatives for prediction validation [50]. We have developed these approaches to serve as a useful way to obtain higher-confidence predictions using all available information and thus we hope that new opportunities will span from this.

\section{Methods}

Heterogeneity of formats and normalization of scores. Prior to the combination of databases, the heterogeneity of information as well as storing formats must be taken into account. One of such heterogeneities concerns transcript-wise or gene-wise identification of interactions. In order to unify those from different databases, we converted transcript-wise predictions to the gene level. Similarly, we unified gene and microRNA names by translating from one nomenclature to another by using dictionaries. We have used a dictionary between microRNA names from mirbase (ftp://mirbase.org/pub/ mirbase/CURRENT/aliases.txt.gz), and translations between different gene names retrieved from Ensembl Biomart. Finally, with the aim of unifying the different scores used in each database, we normalized each score by scaling them to range between 0 and 1 , being 1 the highest confidence for the interaction. Another normalization strategy was tested to eliminate the effect of high dense scoring ranges in the original databases. To that end, the scores from each database were substituted by one minus their cumulative density function (cdf) evaluated at the location of the score. In other words, the scores from each database were sorted in an increasing manner and its new score was calculated as one minus their rank, and then divided by the number of interactions. The results with this normalization method do not show any improvement on the approaches we propose here, which reflects their robustness.

Measuring the reliability of databases. As a measure of the reliability of the databases, a similar measuring approach to that described in [51] was used. In brief, the reliability is measured as the enrichment in experimentally validated interactions and it is determined from the hypergeometric distribution. Let's assume that $\mathrm{E}$ is a set that includes all the interactions predicted by any of the databases with any score. Few interactions in $\mathrm{E}$ have been experimentally validated. Each of the databases provides a set of scored interactions. For each database, we sort the interactions according to their scores and we run one hypergeometric test for each threshold in the scores. Finally, we determine the highest enriched set of interactions by selecting the threshold associated to the lowest $\mathrm{p}$-value. The $\mathrm{p}$-value is an indicative of the enrichment in experimentally validated interactions. The lower the $\mathrm{p}$ value the more enriched the selected set will be. Since the p-values are very small, it is likely to have round-off errors and therefore the approximation suggested in [52] was used. Results are included in Table 3.

Combination of experimental databases. The experimentally validated interactions have been used here as a gold standard to measure the reliability of predicted interactions. In this work, the union of all the experimentally validated interactions has been considered. A brief description of the databases used can be found in Table 1.

Evaluating the performance. The comparison of the performance of our both approaches and the predictive databases was carried out by using the ROC and the Precision curves. The set of the parameters of the ROC curve, number of true positives (TP), number of false positives (FP), number of true negatives (TN) and number of false 
negatives (FN) have been determined by considering the experimentally validated interactions. In fact, an interaction will be considered to be a: TP in case it has been predicted and validated, a TN in case it has neither been predicted nor validated and a FP or FN in case it has either been predicted and not validated or it has been validated but not predicted.

In machine learning, the area under ROC Curve (AUC) is one of the most widely used approaches for performance measurements. In the ROC curve, the TPR (True Positive Rate) is plot against the FPR (False Positive Rate). The sensitivity or True Positive Rate (TPR) is calculated as: $\mathrm{TPR}=\mathrm{TP} /(\mathrm{TP}+\mathrm{FN})$ while 1 -specificity of False Positive Rate $(\mathrm{FPR})$ is calculated as FPR $=\mathrm{FP} /(\mathrm{FP}+\mathrm{TN})$. Each point in a ROC curve is obtained by setting different threshold values to the normalized scores. This threshold is varied in decreasing order, from 1 (most stringent) down to 0 (more relaxed). Figure 1 shows the ROC curve for all predictive algorithms used in this study as well as the two integrating approaches described here.

This evaluation approach has some caveats. First, not all real interactions have been experimentally validated. And secondly, in general, databases with experimentally validated interactions do not include tested and not validated interactions. Therefore, some of the false positives and almost all true negatives will be erroneously labeled. This implies that the ROC curve can produce to unavoidable misleading evaluations.

An alternative approach could be to use the PrecisionRecall curve (PR). In this curve, the Precision $=\mathrm{TP} /(\mathrm{TP}+$ FP) is plotted against the TPR, also known as recall. However, it has been shown that an approach that dominates in the ROC space it also dominates in the PR space [53]. Hence, the Precision-Recall curve also suffers from the same restrictions.

To cope with this situation and to complement the information from the ROC curve, we introduce the Precision curve. For every database, the normalized scores are sorted in descending order and the accumulated precision values are determined. The resulting curve shows the fraction of the predicted interactions that have been experimentally validated versus the number of predicted interactions. This approach is not immune to the potential missing information since there is still a dependency of false positive values, which cannot always be estimated. However, the effect of true negatives is not taken into account, which minimizes an important source of missing information. Figure 2 shows the precision curve for all predicting algorithms as well as for two combination methods described in this manuscript.

Concerning the evaluation of the LRS method using the ROC curve it is important to point out that the model could be overestimated, since the same experimentally validated interactions have been used for both the prediction and the evaluation. However, given that the number of parameters used in the model is much smaller than the number of interactions, this overestimation is not expected to be large. In fact, using glmnet [54] the $\mathrm{R}$ package that internally performs cross-validation to find out the values of the regressors, we got a very similar AUC ( 0.84 using all the data vs. 0.836 using cross-validation) (see supplementary section 3 ).

Approach \#1. Weighted scoring by precision (WSP). The WSP method combines the scores of each interaction in different databases by calculating a weighted sum of their normalized individual scores (see Figure 3.a)). The weights are included to consider that the scoring methods used in different databases are not equally reliable. To this aim, the interactions in each database are sorted from the best to the worst and then, the accumulated precision $(\mathrm{TP} /(\mathrm{TP}+\mathrm{FP}))$ for each of the positions in the sorted list (each interaction) is determined. The accumulated precision for one interaction takes into account the number of TPs and FPs from the first interaction. In order to account for the reliability of the database, the precision value of each interaction is corrected by subtracting the expected precision value for the database, obtaining positive values only for those interactions that are not performing similarly to randomly selected ones. Each new integrated score is then calculated as the sum of each individual score from each database multiplied by the precision of that interaction in the specific database (weight).

With this method, highly re-scored interactions will be those that: a) have been highly scored in individual databases, b) are more likely to be experimentally validated and c) have been predicted with high score in many of the predictive databases. This global score is robust to the incorporation of low-performing scores, i.e. databases with low-performing score will not drag down good scoring ones.

Approach \#2. Logistic Regression combined Scoring (LRS). The LRS method assumes that the higher the probability of an interaction of being experimentally validated, the higher its reliability is. This method, first, determines the probability of each interaction in each database of being experimentally validated and then, combines them to get for each interaction a single probability by using a logistic regression model. The steps, detailed in Figure 3.b) are the following: 1) interactions in each database are ranked according to their scores, then 2) the scored list of each database is divided into a number of bins for which the fraction of the number of experimentally-validated interactions is determined, 3) the obtained set of points is interpolated using smoothing splines and 4) finally, these new scores are combined using a logistic regression model.

Figure S1 of the supplementary materials shows the interpolating splines for each of the databases that in 


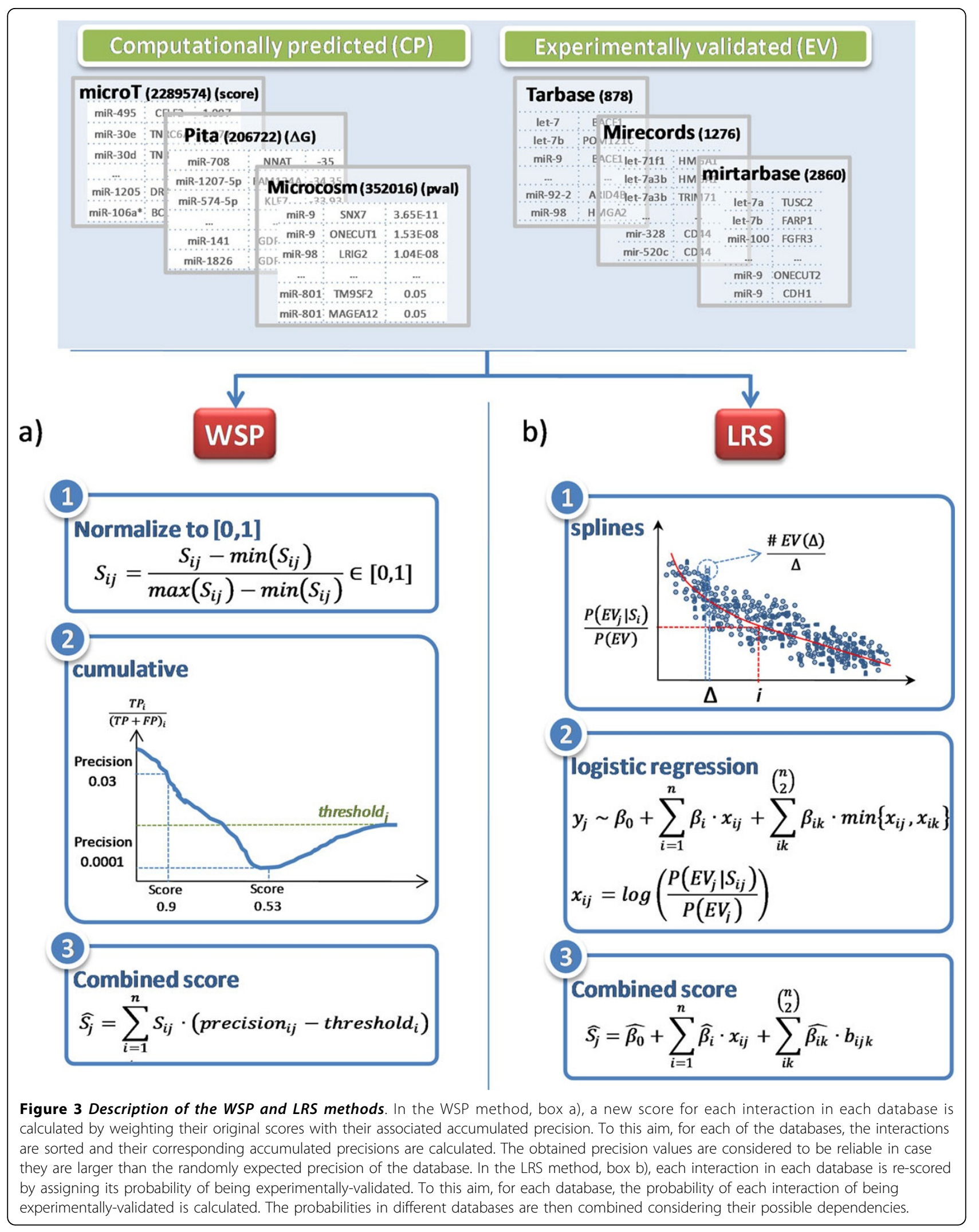


turn, provide the probability of each interaction in a database of being experimentally validated. The proposed logistic regression model is equivalent under some conditions to a probabilistic model (see supplementary material). The logistic model includes crossterms across the databases to accommodate possible redundancies in their information.

miRNA-mRNA database: $\mathbf{m}^{3}$ RNA. The combined databases obtained with both methods have been included into a web page (also accessible via webservices). This webservice also includes the computationally predictions interactions from the different databases used here. miRBase and Ensembl names have been used as the reference name for miRNAs and genes and transcripts respectively.

The database has been implemented using postgreSQL, and all operations and accessions are managed by a Ruby interface. This interface is connected to a SOAP webservices server to provide a remote programmatic access allowing read only operations. Users can access the information within the webservice by providing the organism and a list of miRNAs and/or genes. Data is returned in table format with: the combined databases, names of the miRNAs and mRNAs involved in each interaction, experimental information in case available and, the normalized scores and precisions for every predictive algorithm.

To access the information in a more friendly manner, we have created a website on top of the webservices using the web application framework Ruby on Rails. Further information can be found in the "Help" section of the web page. The database is available at http:// m3rna.cnb.csic.es

\section{Availability of supporting data}

$M^{3}$ RNA website is freely available on the web at http:// m3rna.cnb.csic.es/

\section{Additional material}

Additional File 1: Contains a table with a brief description of methods for the combination of miRNA-mRNA interactions from different databases, figures of the alternative and discarded scoring normalization method for predictive databases, a mathematical description of the LRS method, cross validation results to test overfitting of the model and comparison with other integration methods.

\section{Competing interests}

The authors declare that they have no competing interests.

\section{Authors' contributions}

DTM and ISC developed the code and simulations for the WSP method. AM developed the code and simulations for LRS method. DJMH collected information and data from all available predicting and experimental interaction databases. COSS provided insights in the statistical evaluations. DTM, ISC, DJMH and AM developed and implemented all methodologies described in this study. DTM developed the $m^{3} R N A$. APM and AR conceived the idea, designed experiments and supervised the project. All of the authors participated in the redaction, read and approved the final manuscript.

\section{Acknowledgements}

This work was supported by the Spanish Minister of Science and Innovation with grant [BIO2013-48028-R], the Government of Madrid (CAM) with grant [P2010/BMD-2305], the PRB2-ISCIII, platform supported by grant PT13/ 0001 and the Children Tumor Foundation. AM acknowledges his PhD fellowship of the Basque Country Government. DJMH acknowledges his fellowship from Obra Social "La Caixa". COSS acknowledges the Ramon y Cajal research program. The authors acknowledge Integromics (http://www. integromics.com) for providing the Integromics OmicsOffice ${ }^{\mathrm{TM}}$ product for Tibco Spotfire to generate the images.

\section{Declarations}

The authors acknowledge support of the publication fee by the CSIC Open Access Publication Support Initiative through its Unit of Information Resources for Research (URICI).

This article has been published as part of BMC Genomics Volume 15 Supplement 10, 2014: Proceedings of the 25th International Conference on Genome Informatics (GIW/ISCB-Asia): Genomics. The full contents of the supplement are available online at http://www.biomedcentral.com/ bmcgenomics/supplements/15/S10.

\section{Authors' details}

${ }^{1}$ National Center for Biotechnology-CSIC. Darwin 3. 28049, Madrid, Spain. ${ }^{2}$ CEIT and TECNUN, University of Navarra, San Sebastián, Spain.

Published: 12 December 2014

\section{References}

1. Lee RC, Feinbaum RL, Ambros V: The C. elegans heterochronic gene lin-4 encodes small RNAs with antisense complementarity to lin-14. Cell 1993, 75:843-54

2. Brodersen $P$, Voinnet $O$ : Revisiting the principles of microRNA target recognition and mode of action. Nat Rev Mol Cell Biol 2009, 10:141-8.

3. Bartel DP: MicroRNAs: genomics, biogenesis, mechanism, and function. Cell 2004, 116:281-97.

4. Place RF, Li L-C, Pookot D, Noonan EJ, Dahiya R: MicroRNA-373 induces expression of genes with complementary promoter sequences. Proc Natl Acad Sci USA 2008, 105:1608-13.

5. Brennecke J, Stark A, Russell RB, Cohen SM: Principles of microRNA-target recognition. PLoS Biol 2005, 3:e85.

6. Houbaviy HB, Murray MF, Sharp Pa: Embryonic stem cell-specific MicroRNAs. Dev Cell 2003, 5:351-8.

7. Cheng AM, Byrom MW, Shelton J, Ford LP: Antisense inhibition of human miRNAs and indications for an involvement of miRNA in cell growth and apoptosis. Nucleic Acids Res 2005, 33:1290-7.

8. Zhao Y, Ransom JF, Li A, Vedantham V, von Drehle M, Muth AN, Tsuchihashi T, McManus MT, Schwartz RJ, Srivastava D: Dysregulation of cardiogenesis, cardiac conduction, and cell cycle in mice lacking miRNA1-2. Cell 2007, 129:303-17.

9. Hwang HW, Mendell JT: MicroRNAs in cell proliferation, cell death, and tumorigenesis. $\mathrm{Br} J$ Cancer 2006, 94:776-80.

10. Hansen $T$, Olsen $L$, Lindow $M$, Jakobsen $K D$, Ullum $H$, Jonsson $E$, Andreassen O a, Djurovic S, Melle I, Agartz I, Hall H, Timm S, Wang AG, Werge T: Brain expressed microRNAs implicated in schizophrenia etiology. PLoS One 2007, 2:e873.

11. Lewis BP, Shih I, Jones-Rhoades MW, Bartel DP, Burge CB: Prediction of mammalian microRNA targets. Cell 2003, 115:787-98.

12. Doench JG, Sharp P a: Specificity of microRNA target selection in translational repression. Genes Dev 2004, 18:504-11.

13. Kertesz M, lovino N, Unnerstall U, Gaul U, Segal E: The role of site accessibility in microRNA target recognition. Nat Genet 2007, 39:1278-84

14. Grimson A, Farh KKH, Johnston WK, Garrett-Engele P, Lim LP, Bartel DP: MicroRNA targeting specificity in mammals: determinants beyond seed pairing. Mol Cell 2007, 27:91-105. 
15. Reyes-Herrera PH, Ficarra E: One decade of development and evolution of microRNA target prediction algorithms. Genomics Proteomics Bioinformatics 2012, 10:254-63.

16. Kozomara A, Griffiths-Jones S: miRBase: integrating microRNA annotation and deep-sequencing data. Nucleic Acids Res 2011, 39(Database):D152-7.

17. Dweep H, Sticht C, Pandey P, Gretz N: miRWalk-database: prediction of possible miRNA binding sites by "walking" the genes of three genomes. J Biomed Inform 2011, 44:839-47.

18. Xiao F, Zuo Z, Cai G, Kang S, Gao X, Li T: miRecords: an integrated resource for microRNA-target interactions. Nucleic Acids Res 2009, 37(Database):D105-10.

19. Sethupathy P, Corda B, Hatzigeorgiou AG: TarBase: A comprehensive database of experimentally supported animal microRNA targets. RNA 2006, 12:192-7.

20. Hsu SD, Lin FM, Wu WY, Liang C, Huang WC, Chan WL, Tsai WT, Chen GZ, Lee CJ, Chiu CM, Chien CH, Wu MC, Huang CY, Tsou AP, Huang HD: miRTarBase: a database curates experimentally validated microRNAtarget interactions. Nucleic Acids Res 2011, 39(Database):D163-9.

21. Yang JH, Li JH, Shao P, Zhou H, Chen YQ, Qu LH: starBase: a database for exploring microRNA-mRNA interaction maps from Argonaute CLIP-Seq and Degradome-Seq data. Nucleic Acids Res 2011, 39(Database):D202-9.

22. Chi SW, Zang JB, Mele A, Darnell RB: Argonaute HITS-CLIP decodes microRNA-mRNA interaction maps. Nature 2009, 460:479-86.

23. Enright AJ, John B, Gaul U, Tuschl T, Sander C, Marks DS: MicroRNA targets in Drosophila. Genome Biol 2003, 5:R1.

24. Thadani R, Tammi MT: MicroTar: predicting microRNA targets from RNA duplexes. BMC Bioinformatics 2006, , 7 Suppl 5: S20.

25. Ye W, Lv Q, Wong C-KA, Hu S, Fu C, Hua Z, Cai G, Li G, Yang BB, Zhang Y: The effect of central loops in miRNA:MRE duplexes on the efficiency of miRNA-mediated gene regulation. PLoS One 2008, 3:e1719.

26. Ivo L, Hofacker WFPFSLSBMTPS: Fast Folding and Comparison of RNA Secondary Structures (The Vienna RNA Package)

27. Rehmsmeier M, Steffen $P$, Hochsmann M, Giegerich R: Fast and effective prediction of microRNA/target duplexes. RNA 2004, 10:1507-17.

28. Laganà A, Forte S, Russo F, Giugno R, Pulvirenti A, Ferro A: Prediction of human targets for viral-encoded microRNAs by thermodynamics and empirical constraints. J RNAi Gene Silencing 2010, 6:379-85.

29. Mendoza MR, da Fonseca GC, Loss-Morais G, Alves R, Margis R, Bazzan ALC: RFMirTarget: Predicting Human MicroRNA Target Genes with a Random Forest Classifier. PLoS One 2013, 8:e70153.

30. Mitra R, Bandyopadhyay S: MultiMiTar: A Novel Multi Objective Optimization based miRNA-Target Prediction Method. PLoS One 2011, 6 e24583.

31. Bandyopadhyay S, Member S, Saha S, Member S, Maulik U, Deb K: A Simulated Annealing-Based Multiobjective Optimization Algorithm: AMOSA. 2008, 12:269-283.

32. Yousef M, Jung $S$, Kossenkov A V, Showe LC, Showe MK: Naïve Bayes for microRNA target predictions-machine learning for microRNA targets. Bioinformatics 2007, 23:2987-92.

33. Gaidatzis D, van Nimwegen E, Hausser J, Zavolan M: Inference of miRNA targets using evolutionary conservation and pathway analysis. BMC Bioinformatics 2007, 8:69.

34. Maragkakis $M$, Reczko $M$, Simossis $\vee$ a, Alexiou $P$, Papadopoulos $G L$, Dalamagas T, Giannopoulos G, Goumas G, Koukis E, Kourtis K, Vergoulis T, Koziris N, Sellis T, Tsanakas P, Hatzigeorgiou aG: DIANA-microT web server: elucidating microRNA functions through target prediction. Nucleic Acids Res 2009, 37(Web Server):W273-6.

35. Griffiths-Jones S, Saini HK, van Dongen S, Enright AJ: miRBase: tools for microRNA genomics. Nucleic Acids Res 2008, 36(Database):D154-8.

36. Betel D, Wilson M, Gabow A, Marks DS, Sander C: The microRNA.org resource: targets and expression. Nucleic Acids Res 2008, 36(Database): D149-53.

37. Lewis BP, Burge CB, Bartel DP: Conserved seed pairing, often flanked by adenosines, indicates that thousands of human genes are microRNA targets. Cell 2005, 120:15-20.

38. Wang $X$ : miRDB: a microRNA target prediction and functional annotation database with a wiki interface. RNA 2008, 14:1012-7.

39. Wang X, El Naqa IM: Prediction of both conserved and nonconserved microRNA targets in animals. Bioinformatics 2008, 24:325-32.
40. Sethupathy $P$, Megraw M, Hatzigeorgiou AG: A guide through present computational approaches for the identification of mammalian microRNA targets. Nat Methods 2006, 3:881-6.

41. Krek A, Grün D, Poy MN, Wolf R, Rosenberg L, Epstein EJ, MacMenamin P, da Piedade I, Gunsalus KC, Stoffel M, Rajewsky N: Combinatorial microRNA target predictions. Nat Genet 2005, 37:495-500.

42. Kuhn DE, Martin MM, Feldman DS, Terry A V, Nuovo GJ, Elton TS: Experimental validation of miRNA targets. Methods 2008, 44:47-54.

43. Coronnello C, Benos PV: ComiR: combinatorial microRNA target prediction tool. Nucleic Acids Res 2013, 1-6.

44. Gamazon ER, Im HK, Duan S, Lussier YA, Cox NJ, Dolan ME, Zhang W: Exprtarget: an integrative approach to predicting human microRNA targets. PLOS One 2010, 5:e13534.

45. DeConde RP, Hawley S, Falcon S, Clegg N, Knudsen B, Etzioni R: Combining results of microarray experiments: a rank aggregation approach. Stat Appl Genet Mol Biol 2006, 5:Article15.

46. Yue D, Guo M, Chen Y, Huang Y: A Bayesian decision fusion approach for microRNA target prediction. BMC Genomics 2012, 13(Suppl 8):S13.

47. Huang JC, Frey BJ, Morris QD: Comparing sequence and expression for predicting microRNA targets using GenMiR3. Pac Symp Biocomput 2008, 52-63.

48. Stingo FC, Chen YA, Vannucci M, Barrier M, Mirkes PE: A Bayesian graphical modeling approach to microrna regulatory network inference. Ann Appl Stat 2010, 4:2024-2048.

49. Fawcett T: An introduction to ROC analysis. Pattern Recogn Lett 2006, 27:861-874.

50. Jacobsen A, Silber J, Harinath G, Huse JT, Schultz N, Sander C: Analysis of microRNA-target interactions across diverse cancer types. Nat Struct Mol Biol 2013, 20:1325-1332.

51. Muniategui A, Nogales-Cadenas R, Vázquez $M, L$ Aranguren $X$, Agirre $X$, Luttun A, Prosper F, Pascual-Montano A, Rubio A: Quantification of miRNAmRNA interactions. PLoS One 2012, 7:e30766.

52. Ling RF, Pratt JW: The Accuracy of Peizer Approximations to the Hypergeometric Distribution, with Comparisons to Some other Approximations. J Am Stat Assoc 1984, 79:49-60.

53. Davis J, Goadrich M: The relationship between Precision-Recall and ROC curves. Proc 23rd Int Conf Mach Learn ICML 06 2006, 10:233-240, [ICML '06]

54. Friedman J, Hastie T, Tibshirani R: Regularization Paths for Generalized Linear Models via Coordinate Descent. J Stat Softw 2010, 33:1-22.

doi:10.1186/1471-2164-15-S10-S2

Cite this article as: Tabas-Madrid et al:: Improving miRNA-mRNA interaction predictions. BMC Genomics 2014 15(Suppl 10):S2.

\section{Submit your next manuscript to BioMed Central and take full advantage of:}

- Convenient online submission

- Thorough peer review

- No space constraints or color figure charges

- Immediate publication on acceptance

- Inclusion in PubMed, CAS, Scopus and Google Scholar

- Research which is freely available for redistribution

Submit your manuscript at www.biomedcentral.com/submit
C Biomed Central 